\title{
Optimum phase shift applied to weighted-polarization MIMO antenna in various use scenarios
}

\author{
Kazuhiro Honda ${ }^{\text {a) }}$ and Kun Li \\ Graduate School of Engineering, Toyama University, \\ 3190 Gofuku, Toyama-shi, Toyama 930-8555, Japan \\ a)hondak@eng.u-toyama.ac.jp
}

\begin{abstract}
This paper presents a method to achieve the maximum MIMO channel capacity using a weighted-polarization MIMO antenna. Particular emphasis is placed on the way to adjust the phase shift value between the antenna elements to an optimum value in accordance with the variation of cross polarization power ratio $(X P R)$ and the antenna inclination angle. Both the measurement and analytical results show that using the optimum phase shift value, the MIMO channel capacity is increased in comparison with a conventional fixed-phase shift antenna in various conditions.
\end{abstract}

Keywords: weighted-polarization MIMO antenna, phase shift value, MIMO channel capacity, MIMO-OTA

Classification: Antennas and Propagation

\section{References}

[1] D. Gesbert, M. Shafi, D. S. Shiu, P. Smith, and A. Naguib, "From theory to practice: an overview of MIMO space-time coded wireless systems," IEEE J. Sel. Areas Commun., vol. 21, no. 3, pp. 281-302, Apr. 2003. DOI:10.1109/ JSAC.2003.809458

[2] C. Chong, F. Watanabe, K. Kitao, T. Imai, and H. Inamura, "Evolution trends of wireless MIMO channel modeling towards IMT-advanced," IEICE Trans. Commun., vol. E92-B, no. 9, pp. 2773-2788, Sep. 2009. DOI:10.1587/ transcom.E92.B.2773

[3] T. Aoyagi, M. Kim, J. Takada, K. Hamaguchi, and R. Kohno, "Numerical simulations for wearable BAN propagation channel during various human movements," IEICE Trans. Commun., vol. E94-B, no. 9, pp. 2496-2500, Sep. 2011. DOI:10.1587/transcom.E94.B.2496

[4] K. Honda, K. Li, and K. Ogawa, "Weighted-polarization wearable MIMO antenna with three orthogonally arranged dipoles based on RF signal processing," IEICE Trans. Commun., vol. E99-B, no. 1, pp. 58-68, Jan. 2016. DOI:10. 1587/transcom.2015ISP0015

[5] K. Honda, T. Kabeya, K. Karitani, K. Li, K. Ogawa, Y. Koyanagi, H. Sato, and R. Miura, "A polarization-controlled MIMO antenna with an optimum phase shift in accordance with various use scenarios," IEEE AP-S Intl. Symp. Digest, Vancouver, Canada, pp. 1234-1235, July 2015. DOI:10.1109/APS.2015. 7305006

[6] M. Yamazaki, K. Honda, and K. Ogawa, "A high-sensitivity MRC diversity 
antenna for tablet handsets considering the mutual interactions between the holding angle and broadcasting waves polarization," IEICE Trans. Commun., vol. J96-B, no. 9, pp. 991-1000, Sep. 2013.

[7] K. Honda, K. Li, and K. Ogawa, "A calibration method for the OTA testing of active MIMO antennas," IEICE Tech. Report, AP2014-60, pp. 67-72, Jul. 2014.

[8] K. Honda, T. Kabeya, K. Karitani, K. Li, K. Ogawa, Y. Koyanagi, H. Sato, and S. Ueda, "A bilateral MIMO-OTA system for the combined antenna evaluation considering uplink and downlink channels," IEICE Tech. Report, AP2014-74, pp. 13-18, Aug. 2014.

[9] K. Ogawa, H. Iwai, A. Yamamoto, and J. Takada, "Channel capacity of a handset MIMO antenna influenced by the effects of 3D angular spectrum, polarization, and operator,” IEEE AP-S Int. Symp. Digest, pp. 153-156, July 2006. DOI:10.1109/APS.2006.1710476

\section{Introduction}

Ultra high-speed and high-capacity mobile communication is coming as a result of the development of LTE-Advanced and Beyond 4G cellular systems using multiple-input multiple-output (MIMO) technique [1]. Since a small cell system is taken into consideration in future ultra high-capacity mobile communications [2], the cross polarization power ratio $(X P R)$ in a radio wave propagation environment will vary significantly. Further, the polarization characteristics of a wearable antenna will also change easily due to the antenna inclination caused by the dynamic motion of an operator [3].

In our previous study [4], a weighted-polarization wearable MIMO antenna was proposed, where two of the three antenna elements are combined using weight functions, which considers $X P R$ and antenna inclination angle as key parameters. However, the phase shift value between the two elements was fixed to 90 degrees considering the balance of influence on the received signal power when the antenna inclination angle is varied from -90 to 90 degrees. To achieve the maximum MIMO channel capacity in various use scenarios, the optimum phase shift value needs to be investigated.

This paper presents an investigation of the optimum phase shift value applied to the weighted-polarization MIMO antenna [5]. By adjusting a phase shift value between the antenna elements to an optimum value in accordance with various use scenarios, the MIMO channel capacity is increased significantly in comparison with a conventional fixed-phase shift antenna described in [4].

\section{Weighted-polarization MIMO antenna}

Fig. 1(a) shows the configuration of the weighted-polarization MIMO antenna [4]. The antenna is comprised of three orthogonal dipole antennas $\left(A_{x}, A_{y}, A_{z}\right)$. When the antenna is rotated by an operator, two of the three dipole antennas are selected using two switches $\left(\mathrm{SW}_{1}, \mathrm{SW}_{2}\right)$.

The selection of elements is determined by the use scenarios of a tablet terminal, as shown in Fig. 1(b). In the case of the left figure in Fig. 1(b), the element $A_{y}$ is not selected because $A_{y}$ remains unchanged in the horizontal 
configuration that receives the horizontally polarized component all the time and thus $\mathrm{A}_{\mathrm{y}}$ does not have the ability to provide a varied polarized component. In a similar way, the element $A_{x}$ is not selected in the case of the right figure in Fig. 1(b) because $A_{x}$ remains unchanged in the horizontal configuration.

The received signals $\left(s_{V}, s_{H}\right)$ are multiplied by the weight functions $\left(W_{V}{ }^{\prime}, W_{H}{ }^{\prime}\right)$. Thus, the signal at the output port ( $a$ ) of the antenna is expressed by Eqs. (1)-(5).

$$
\begin{aligned}
a & =\frac{W_{V}^{\prime}}{\sqrt{W_{V}^{\prime 2}+W_{H}^{\prime 2}}} s_{V}+\frac{W_{H}^{\prime}}{\sqrt{W_{V}^{\prime 2}+W_{H^{\prime 2}}}} s_{H} e^{j \tau} \\
W_{V}^{\prime} & =W_{V}|\cos \alpha|+W_{H}|\sin \alpha| \\
W_{H}^{\prime} & =W_{V}|\sin \alpha|+W_{H}|\cos \alpha| \\
W_{V} & =\sqrt{\frac{X P R}{1+X P R}} \\
W_{H} & =\sqrt{\frac{1}{1+X P R}}
\end{aligned}
$$

In Fig. 1(a), a variable phase shifter is used in order to realize the phase shift value $\tau$ between the two elements, as denoted by Eq. (1). In our previous study [4], the phase shift value $\tau$ in Eq. (1) was fixed to 90 degrees considering the balance of influence on the received signal power when the antenna tilt angle $\alpha$ is inclined from -90 to 90 degrees.

However, when the antenna is mounted on a tablet terminal, as shown in Fig. 1(b), the inclination angle $\alpha$ is changed mainly in a range from 0 to 90 degrees, which means that $\alpha$ is not a negative value in this situation. In this case of $\alpha>0$, the polarization combination mechanism of the weighted-polarization MIMO antenna is limited to the case as shown in Fig. 1(c).

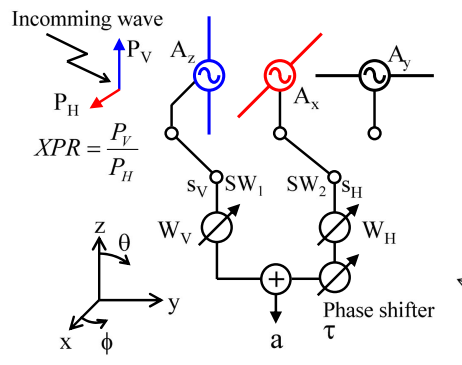

(a) Antenna configuration

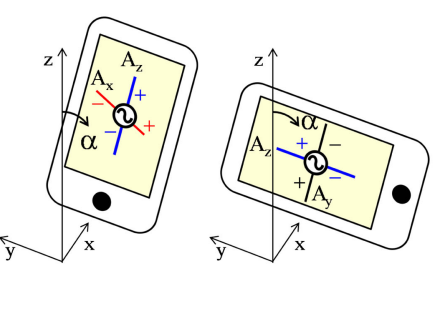

(b) Use scenarios

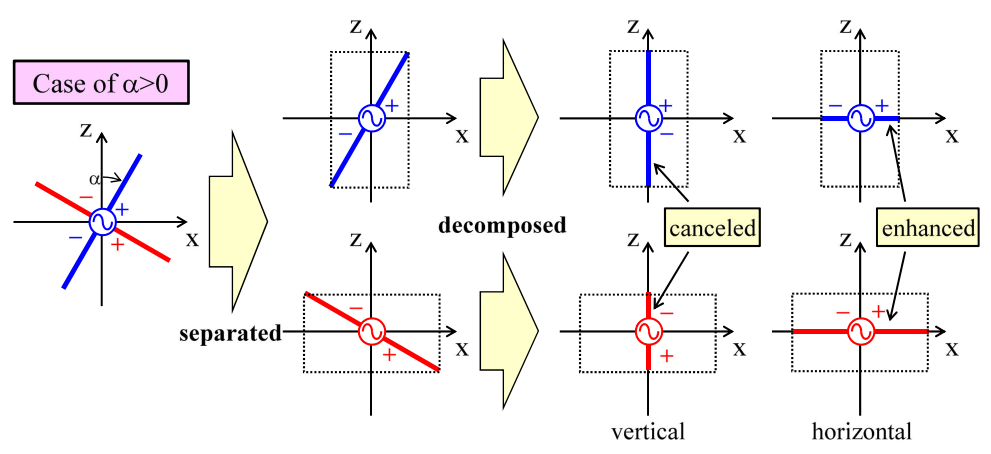

(c) Polarization combination mechanism

Fig. 1. Weighted-polarization MIMO antenna. 
In Fig. 1(c), the weighted-polarization MIMO antenna is separated into two crossed dipole antennas, which can be decomposed into vertical and horizontal components, respectively. Thus, the polarization components distributed in the vertical direction are canceled with each other due to the opposite voltages applied to the antenna exciting terminals whereas the polarization components in the horizontal direction are enhanced because of the common voltages. Therefore, the optimum phase shift value between the two elements is investigated in this paper.

If the phase shift value $\tau$ is set to 0 degree, the vertical and horizontal polarization component is canceled and enhanced, respectively. Therefore, the radiation pattern of the weighted-polarization MIMO antenna is suitable for the environment where the XPR expressed in decibel is negative. On the other hand, if the phase shift value $\tau$ is set to 180 degrees, the vertical and horizontal polarization component is enhanced and canceled, respectively. Therefore, the radiation pattern of the antenna is suitable for the environment where the XPR is positive. Hence, the optimum phase shift value is varied according to different propagation environments of $X P R$. Base on the abovementioned mechanism, the optimum phase shift value according to $X P R$ and antenna inclination angle can be determined.

\section{Radiation characteristics}

In our previous study [6], it is found that the average holding angle of a tablet terminal is 55 degrees. Based on this statistical investigation, the measurements of radiation pattern and channel capacity using the weighted-polarization MIMO antenna are carried out in this paper.

Fig. 2(a) shows the cofiguration of the $2 \times 2$ MIMO antenna. Fig. 2(b) shows the photograph of the array antenna in an anechoic chamber. The two orthogonal half-wavelength dipole antennas were arranged as a MIMO array in the y-direction. Based on the method of the element selection described in Sect. 2, the experimental setup comprised of a two-element crossed dipole antenna is used to represent the state when the two elements of the proposed antenna are selected by the switches $\mathrm{SW}_{1}$ and $\mathrm{SW}_{2}$, as shown in Fig. 1(a). The frequency for the measurements was $2 \mathrm{GHz}$. The distance between the two array elements was set to $7.5 \mathrm{~cm}$.

The signal combining of the two elements based on Eq. (1) is realized by the use of the RF processing unit, which comprises metalic coaxial cables, variable attenuators and a power divider, as shown in Fig. 2(c). The phase shift value $\tau$ in Eq. (1) is realized by adjusting the length of metalic coaxial cables. In the measurement, we performed a calibration to compensate the insertion loss of attenuators [7]. Fig. 2(d), (e) and (f) show the radiation pattern in the xy-plane with respect to the variation of phase shift value $\tau$ when the XPR in the weight function is set to $10 \mathrm{~dB}$. The blue line indicates the $E_{\theta}$-component while the red line indicates the $E_{\phi}$-component. We carried out an analytical simulation using the method of moments.

As can be seen in Fig. 2(d), (e) and (f), the measured radiation patterns using the weighted-polarization MIMO antenna are in good agreement with the analytical results as a function of the phase shift value $\tau$. When $\tau$ is set to 0 and 180 degrees, 


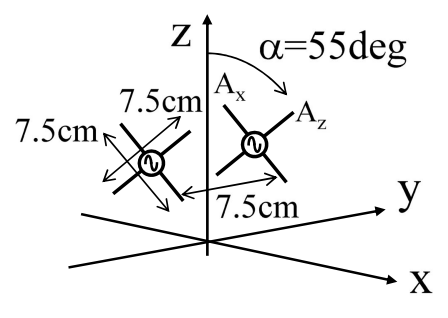

(a) Configuration

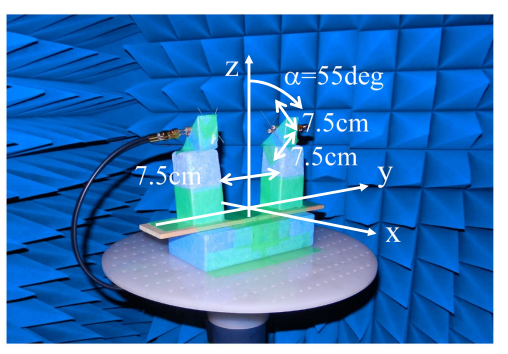

(b) Photograph

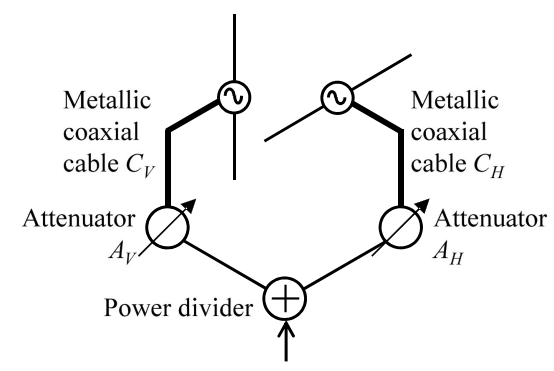

(c) Setup for realizing the weight function
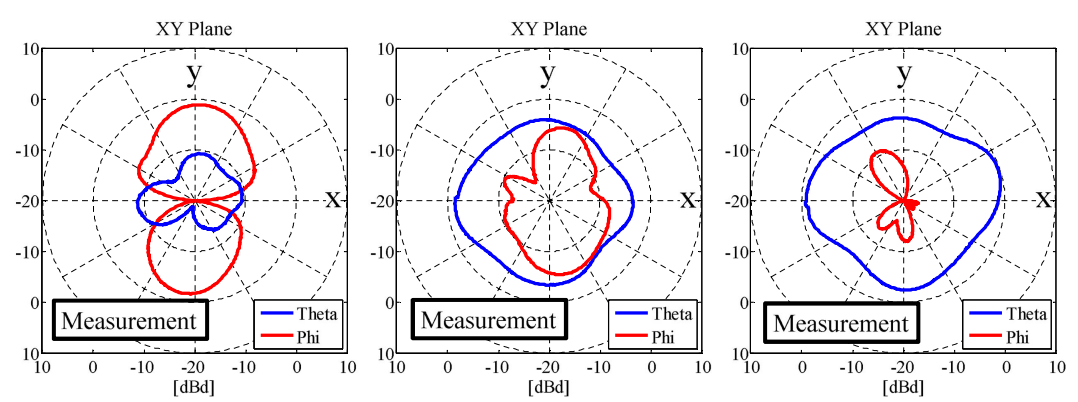

(d1) measurement

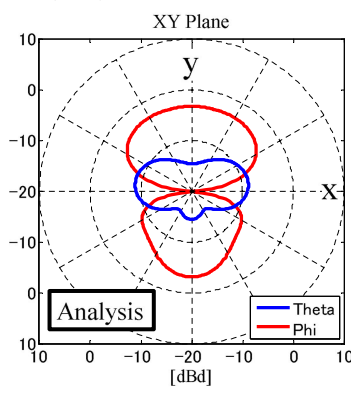

(e1) measurement

(f1) measurement
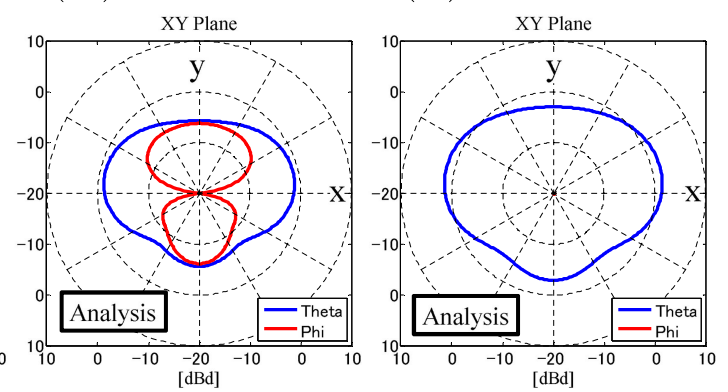

(d2) analysis

(e2) analysis

(f2) analysis

(d) $\tau=0$ deg.

(e) $\tau=90 \mathrm{deg}$.

(f) $\tau=180 \mathrm{deg}$.

Fig. 2. Radiation characteristics.

the $E_{\phi}$ and $E_{\theta}$ components are enhanced, as shown in Fig. 2(d) and (f), respectively. In this manner, the weighted-polarization MIMO antenna provides appropriate radiation patterns in accordance with $X P R$, the antenna inclination angle and phase shift value.

\section{$42 \times 2$ MIMO channel capacity}

The MIMO channel capacity of a $2 \times 2$ weighted-polarization MIMO antenna shown in Fig. 2(b) was measured using a MIMO-OTA (over-the-air) apparatus [8]. 
Fig. 3(a) shows the measurement setup of the weighted-polarization MIMO antenna, which is placed at the center of the spatial fading emulator.

Fig. 3(b) indicates the measured and analytical results of $2 \times 2$ MIMO channel capacity with the phase shift value $\tau$ varied from 0 to 180 degrees when XPR is set to $10 \mathrm{~dB}$ and the antenna inclination angle $\alpha$ was fixed at 55 degrees. The $S N R$ of the incident wave was set to $30 \mathrm{~dB}$. The incident wave around the MIMO antenna was set to a uniform angular power spectra in the horizontal plane. In a similar way used in the measurement of the radiation pattern mentioned in Sect. 3, a calibration of the MIMO-OTA system was carried out [7]. In Fig. 3(b), the symbol $\bullet$ denotes the measured results of the $2 \times 2$ MIMO channel capacity using the weightedpalarization MIMO antenna. The analytical results are calculated by the Monte Carlo simulation [9], as shown by the solid black line.

As can be seen in Fig. 3(b), the measured results are in good agreement with the analytical outcomes. Further, when the phase shift value $\tau$ is set to 180 degrees, a $1.9 \mathrm{bits} / \mathrm{s} / \mathrm{Hz}$ improvement of the channel capacity is achieved in comparison with the conventional 90-degree phase shift antenna. This is because the radiation pattern of the antenna has a large desired vertical polarization component, as shown in Fig. 2(f), and this benefits in a propagation environment when $X P R$ is $10 \mathrm{~dB}$. On the basis of the abovementioned considerations, we conclude that the optimum value of phase shift is 180 degrees when $X P R$ is $10 \mathrm{~dB}$ and the antenna inclination angle is 55 degrees.

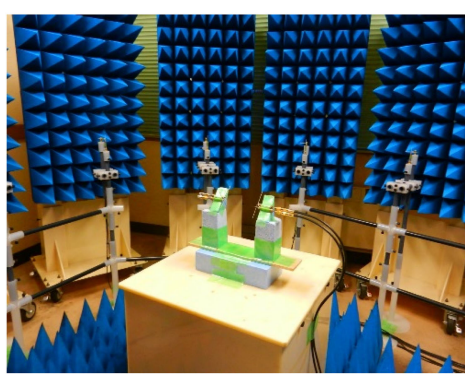

(a) Measurement setup

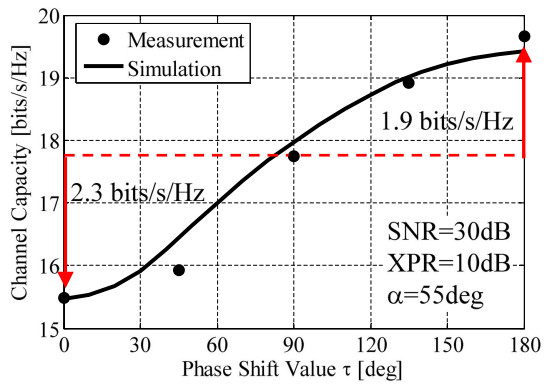

(b) Relationship between phase shift value and channel capacity

Fig. 3. $2 \times 2$ MIMO channel capacity.

\section{Conclusion}

This paper presents a method to achieve the maximum MIMO channel capacity using a weighted-polarization MIMO antenna. By adjusting the optimum phase shift value in accordance with various use scenarios, the MIMO channel capacity is increased more than $1.9 \mathrm{bits} / \mathrm{s} / \mathrm{Hz}$ in comparison with a conventional fixed-phase shift antenna. Future studies include the derivation of optimum phase shift value in accordance with various use scenarios and XPRs.

\section{Acknowledgments}

